\title{
On the optimality of the Friedman Rule in a New Monetarist Model
}

\author{
Ryoji Hiraguchi \\ The Canon Institute for Global Studies, Tokyo, Japan \\ Keiichiro Kobayashi \\ The Canon Institute for Global Studies, Tokyo, Japan
}

September 24, 2013

※Opinions expressed or implied in the CIGS Working Paper Series are solely those of the author, and do not necessarily represent the views of the CIGS or its sponsor.

※ CIGS Working Paper Series is circulated in order to stimulate lively discussion and comments.

※Copyright belongs to the author(s) of each paper unless stated otherwise. 


\title{
On the optimality of the Friedman Rule in a New
}

\author{
Monetarist Model
}

\author{
Ryoji Hiraguchi *and Keiichiro Kobayashi ${ }^{\dagger}$
}

September 24, 2013

\begin{abstract}
We study a monetary model in which buyers choose search intensity and prices are considered as given in the decentralized market. In doing so, we indicate that the Friedman rule may not be optimal. Buyers' search intensity is excessively low under the rule, because their surplus is less than the social surplus under the price-taking regime. A deviation from the rule increases buyers' surplus and search intensity, and thus raises welfare and output. Our result differs from Lagos and Rocheteau (International Economic Review 2005) in which the pricing mechanism is either bargaining or price-posting and the Friedman rule is optimal.
\end{abstract}

${ }^{*}$ Faculty of Economics, Ritsumeikan University. 1-1-1, Noji-higashi, Kusatsu, Shiga, Japan. Email: rhira@fc.ritsumei.ac.jp. Tel: 81-77-561-4837. Fax: 81-77-561-4837.

${ }^{\dagger}$ Faculty of Economics, Keio University. 2-15-45 Mita, Minato-ku, Tokyo, Japan. Email: kobayasi@econ.keio.ac.jp. Tel:81-3-5418-6703. 


\section{Introduction}

The optimality of the Friedman rule, which advocates setting the nominal interest rate to zero has been an important topic in monetary economics. It is widely known to be optimal in basic monetary models such as the money-in-the-utility function model, the shopping-time model and the cash-credit model. A positive nominal interest rate serves as a distortionary tax on money holdings. Therefore it should be removed and the government should rely on other taxes such as a consumption or labor income tax.

These models are occasionally criticized because the role of money in them is not clear. To overcome this criticism, Kiyotaki and Wright $(1989,1993)$ developed a search theoretic model, in which money functions as medium of exchange. These seminal papers spawned a large body of literature focusing on search theoretic monetary models, such as Aiyagari and Wallace (1991), Trejos and Wright (1995) and Berentsen et al. (2007). Among them, Lagos and Wright (2005, henceforth LW) construct a tractable framework, in which monetary policy is easily analyzed and this framework is now called the "New Monetarist Model" (Williamson and Wright (2010)). In LW, transactions at some markets need money due to the anonymity of traders. Interestingly, they show that the Friedman rule is still optimal.

The Lagos-Wright framework has been extended in several directions. For example, Lagos and Rocheteau (2005, henceforth LR) incorporate endogenous search intensity into LW. They consider two pricing mechanisms, bargaining and price posting with directed search and show that while output may increase with inflation, the optimal monetary policy always follows the Friedman rule. These results indicate that the optimality of the Friedman rule remains robust in modern monetary economics.

We study an extension of LR by assuming that the pricing mechanism is price-taking. Each period is divided into two subperiods, day and night. Transactions in the day market need money since it is decentralized. Furthermore, buyers choose costly search intensity which determines the probability of meeting with sellers. Our model differs from LR only in the pricing mechanism. ${ }^{1}$ However, the small difference changes the optimal monetary

\footnotetext{
${ }^{1}$ Strictly speaking, our model differs from LR in the treatment of buyers and sellers. In LR, the agents
} 
policy and for certain parameter values, the Friedman rule is not optimal.

In our model, the Friedman rule achieves efficient trade: however, the search intensity may be inefficiently low. Efficiency in search intensity requires the marginal cost of search effort to be equal to its marginal benefit, that is, the marginal increase of matching probability multiplied by the total surplus. However, when buyers choose their search effort, they are only concerned about their own surplus, which is strictly less than the total surplus as long as the sellers' cost function is strictly convex. Under a price-taking regime, an infinitesimal rise in inflation from the Friedman rule increases buyers' surplus and search intensity, thus increasing social welfare. Inflation reduces the match surplus; however, the first order effect of this reduction is zero according to the Friedman rule and then it is negligible as long as the inflation rate is small. I addition, we find that deviation from the Friedman rule may increase output.

We reinforce Rocheteau and Wright (2005, henceforth RW) in a model where agents are ex ante identical as in LW. In RW, as in our model, the sellers are subject to the free-entry conditions and the matching probability is endogenously determined. In RW, when the pricing mechanism is price-taking, the Friedman rule achieves efficient trade, but the entry level may be inefficiently low. Due to this inefficiency, a deviation from the rule may improve welfare as in our model. Table 1 compares the existing literature with our paper.

\begin{tabular}{|l|l|l|l|l|}
\hline Paper & Trading mechanism & Welfare & Output & Search structure \\
\hline \multirow{2}{*}{ LW } & Bargaining & Yes & Yes & \\
\hline \multirow{4}{*}{ RW } & Bargaining & Yes & Yes & \multirow{2}{*}{ Free entry } \\
\cline { 2 - 4 } & Competitive search & Yes & - & \\
\cline { 2 - 4 } & Price taking & No & - & \multirow{2}{*}{ Costly search } \\
\hline \multirow{2}{*}{ LR } & Bargaining & Yes & Yes & \\
\cline { 2 - 4 } & Price posting & Yes & No & \\
\hline \multirow{2}{*}{ Ours } & Price taking & No & No & Costly search \\
\hline
\end{tabular}

Table 1: Does the Friedman rule maximize welfare and output?

are heterogeneous, but here the agents are ex-ante homogeneous, and receive idiosyncratic preference shocks. However, this difference is not crucial. 
New monetarist models with price-taking have been introduced by RW and investigated by several authors. Berentsen et al. (2007) incorporate the financial intermediation into LW and indicate that it improves welfare as long as a positive nominal interest rate is set. Ferraris and Watanabe (2008) include collateral secured loans and find that inflation enhances capital accumulation. In both papers, however, the optimality of the Friedman rule is unchanged. ${ }^{2}$ These results suggest that endogenous search intensity substantially modifies the nature of the optimal monetary policy under price-taking. The suboptimality of the Friedman rule is caused by market failure due to nonexistence of a competitive market for search intensity.

This paper is organized as follows. Section 2 describes our model. Section 3 considers the optimal monetary policy. Section 4 presents the conclusions. Proofs are included in the Appendix.

\section{Model}

\section{$2.1 \quad$ Set-up}

The set-up in the model presented is similar to that in LR and LW. Time is discrete and changes from $t=0$ to $+\infty$. There is a continuum of infinitely lived agents with unit measure. Each date is divided into two subperiods, day and night. In each subperiod, a perfectly competitive market opens. The day market is decentralized and the night market is centralized. Agents are price-takers at both day and night markets. Money's role is to facilitate trades in the day market, where buyers and sellers are anonymous. The buyers need money to make payments. Money is divisible and storable, but it is intrinsically useless.

At the beginning of the day market, each agent receives an idiosyncratic preference shock, and the agent becomes a buyer or seller with equal probability $\sigma$ with $\sigma \in(0,1 / 2]$. With probability $1-2 \sigma$, he passes the day market. Once he becomes a buyer, he chooses

\footnotetext{
${ }^{2}$ In Aruoba and Chugh (2010), the rule is not optimal, but this is because lump-sum tax is not available by assumption.
} 
search intensity. The utility cost of search $\psi(e)$ is an increasing function of the search intensity $e$, where $\psi(0)=\psi^{\prime}(0)=0, \psi^{\prime}(e)>0$ and $\psi^{\prime \prime}(e)>0$ if $e>0$ and $\psi^{\prime}(1)=$ $+\infty$. In this set-up, $\mu_{b}=\mu_{s}=\sigma$. The aggregate matching function is $\zeta\left(\bar{e} \mu_{b}, \mu_{s}\right)$ where $\bar{e}$ is the average search intensity of buyers, $\mu_{b}$ is the measure of buyers and $\mu_{s}$ is the measure of the seller. The function $\zeta$ is constant returns to scale, twice continuously differentiable, strictly increasing and strictly concave. We assume that $\zeta(0,1)=\zeta(1,0)=$ 0 and $\lim _{x \rightarrow \infty} \zeta(1, x)=1$. Under these assumptions, the function $\pi(e) \equiv \zeta(e, 1)$ satisfies $\pi^{\prime}(e)>0, \pi(0)=0$ and $\pi^{\prime \prime}(e)<0$. For a buyer with search intensity $e$, the probability of matching with a seller is $e \zeta\left(\bar{e} \mu_{b}, \mu_{s}\right) /\left(\bar{e} \mu_{b}\right)=e \bar{\alpha}$ with $\bar{\alpha} \equiv \pi(\bar{e}) / \bar{e}$. For each seller, the probability of meeting with a buyer $\zeta\left(\bar{e} \mu_{b}, \mu_{s}\right) / \mu_{s}=\pi(\bar{e})=\bar{\alpha} \bar{e}$ is exogenous.

In the day market, the buyer obtains utility $u(q)$ from consuming $q$ units of output. The utility function $u$ is twice continuously differentiable and satisfies $u(0)=0, u^{\prime}(q)>$ $0, u^{\prime \prime}(q)<0$ and $\lim _{q \rightarrow 0} u^{\prime}(q)=+\infty$. The cost function of the seller, $c(q)$ is twice continuously differentiable and satisfies $c(0)=0, c^{\prime}(q)>0$ and $c^{\prime \prime}(q)>0$. Let $s(q)=$ $u(q)-c(q)$ denote the match surplus. In the centralized night market, each agent gets utility $U(x)$ from consuming $x$ units of goods and gets linear disutility $x$ from producing $x$ units of goods. We assume that there exists $q^{*}>0$ such that $u^{\prime}\left(q^{*}\right)=c^{\prime}\left(q^{*}\right)$ and $x^{*}>0$ such that $U^{\prime}\left(x^{*}\right)=1$.

A central bank exists and controls the money supply. The growth rate of the money supply $\tau$ is constant. The money supply in period $t, M_{t}$ satisfies $M_{t}=\tau M_{t-1}$. At the end of period $t$, the agent receives lump-sum money transfers $(\tau-1) M_{t}$.

\section{$2.2 \quad$ Night market}

We focus on the degenerate stationary equilibrium in which the amount of production and consumption by all agents is the same and output is constant. Below, consecutive period variables are indexed by +1 . Let $W(m)$ denote the value function of the individual at night who holds $m$ dollars. Let $V(m)$ denote the value function of the agent at the beginning of each period who holds $m$ dollars. An agent's optimization problem at night is as follows: 


$$
\begin{aligned}
W(m) & =\max _{x, h}\left\{U(x)-h+\beta V_{+1}\left(m_{+1}\right)\right\} \\
\text { s.t. } x & =h+\phi\left(m+(\tau-1) M-m_{+1}\right) .
\end{aligned}
$$

where $\phi$ is the price of money in terms of the general goods. The first order conditions are as follows:

$$
\begin{aligned}
\phi & =\beta V_{+1}^{\prime}\left(m_{+1}\right), \\
U^{\prime}(x) & =1 .
\end{aligned}
$$

Thus, trades in the centralized market are efficient. From the quasi-linearity of the utility function in the night market, the function $W$ is a linear function of the nominal balance, and the envelope conditions are as follows:

$$
W^{\prime}(m)=\phi
$$

For a constant $A$, the function $W$ can be written as $W(m)=\phi m+A$.

\subsection{Day market}

First we investigate the problem of a seller. Let $V_{s}(m)$ denote the value function of a seller who holds $m$ dollars. He takes the matching probability $\pi(\bar{e})=\bar{\alpha} \bar{e}$ as given. With probability $1-\pi(\bar{e})$, the seller passes the day market without meeting buyers. The optimization problem of the seller is as follows:

$$
V^{s}(m)=\bar{\alpha} \bar{e} \max _{q}[-c(q)+W(m+p q)]+(1-\bar{\alpha} \bar{e}) W(m)
$$

where $p$ is the price of the good at the day market. From the linearity of $W$, the seller's value function is written as $V^{s}(m)=\bar{\alpha} \bar{e} \max _{q}\{-c(q)+\phi p q\}+\phi m+W(0)$. The first order conditions on $q$ are as follows:

$$
c^{\prime}(q)=\phi p
$$

and we have

$$
\frac{\partial V^{s}}{\partial m}=\phi
$$


Next we investigate the buyers' problem. The buyer is subject to the cash-in-advance constraint. Let $V^{b}(m)$ denote his value function. From the linearity of $W(m)$, the maximization problem can be simplified as follows:

$$
V^{b}(m)=\max _{e}\left[-\psi(e)+e \bar{\alpha} \max _{p q \leq m}[u(q)-\phi p q]\right]+\phi m+W(0) .
$$

Let $s_{b}^{m}=\max _{p q \leq m}[u(q)-\phi p q]$ denote the buyer's surplus from the match. From Eq. (6), the equilibrium surplus $s_{b}(q) \equiv u(q)-q c^{\prime}(q)$ can be expressed as a function of $q$. The first order conditions are as follows:

$$
\begin{aligned}
& e: \psi^{\prime}(e)=\bar{\alpha} s_{b}^{m}, \\
& q: u^{\prime}(q) \geq \phi p .
\end{aligned}
$$

In Eq. (10), the strict inequality holds only if the cash-in-advance constraint is binding (i.e., $p q=m$ ). Eq. (9) implies that the marginal cost of effort is equal to the marginal increase of matching probability multiplied by the buyer's benefit from the match. The envelope condition is

$$
\frac{\partial V^{b}}{\partial m}=\phi+e \bar{\alpha} \frac{\partial s_{b}^{m}}{\partial m}
$$

The function $V$ satisfies $V(m)=\sigma\left(V^{b}(m)+V^{s}(m)\right)+(1-2 \sigma) W(m)$. We use Eqs. (3), (5), (7) and (11) to obtain the relationship between the nominal interest rate $i=\phi /\left(\beta \phi_{+1}\right)-1$ and the quantities:

$$
i=\sigma e \bar{\alpha} \frac{1}{\phi} \frac{\partial s_{b}^{m}}{\partial m}
$$

Since $s_{b}^{m}$ is non-decreasing with $m$, the nominal interest rate $i$ is non-negative.

A stationary equilibrium allocation is $(p, \phi, e, q)$ where $p$ is the price of the specific goods, $\phi$ is the price of money, $e$ is the buyer's search intensity and $q$ is the volume of trade in the day market. In the equilibrium, average effort $\bar{e}$ is equal to individual effort $e$ and the individual money holdings $m$ is equal to the aggregate money supply $M$. At the stationary equilibrium, $\phi / \phi_{+1}=1+\tau$ and $i=(1+\tau) / \beta-1$. 
Proposition 1 A stationary equilibrium allocation $(p, \phi, e, q)$ satisfies

$$
\begin{aligned}
i & =\sigma \pi(e)\left(\frac{u^{\prime}(q)}{c^{\prime}(q)}-1\right) \geq 0 \\
\frac{e \psi^{\prime}(e)}{\pi(e)} & =s_{b}(q) \\
\phi & =p c^{\prime}(q) \\
\phi & =(1+\tau) \phi_{+1}
\end{aligned}
$$

Here $i=(1+\tau) / \beta$ is the nominal interest rate.

Proof. See Appendix.

Eq. (13) implies that $q=q^{*}$ under the Friedman rule. The function $g(e) \equiv e \psi^{\prime}(e) / \pi(e)$ at the left hand side of Eq. (14) is a strictly increasing function of $e$ since $\pi(e) / e=$ $\zeta(1,1 / e)$ is a decreasing function of $e$, and it satisfies $g(0)=0$ and $g(1)=+\infty$. Using $g$, we can re-write Eq. (14) as $g(e)=s_{b}(q)$. Hence the equilibrium search intensity $e(q) \equiv g^{-1}\left(s_{b}(q)\right)$ can be expressed as a function of $q$. Below, we denote the equilibrium effort under the Friedman rule, $e\left(q^{*}\right)$ as $e^{*}$. The two functions $s_{b}(q)=u(q)-q c^{\prime}(q)$ and $e(q)$ satisfy the following lemma.

Lemma 1 The function $s_{b}(q)$ satisfies i) $s_{b}^{\prime}(0)>0$, ii) $s_{b}^{\prime}\left(q^{*}\right)<0$, iii) $s_{b}^{\prime \prime}(q)<0$ and iv) $s(q)>s_{b}(q)>0$ for all $q \in\left(0, q^{*}\right]$. Furthermore, the effort function $e(q)$ satisfies $e^{\prime}\left(q^{*}\right)<0$.

Proof. See the Appendix.

Lemma 1 implies that $s_{b}(q)$ and $e(q)$ have inverted U-shapes. Therefore, the stationary equilibrium may not be unique. Below, we assume that the stationary equilibrium is unique. Later in a parametric example, we obtain a condition to ensure equilibrium uniqueness. From Eq. (13) the nominal interest rate can be expressed as a function of $q$ :

$$
i=\sigma \pi(e(q))\left(u^{\prime}(q) / c^{\prime}(q)-1\right)
$$

In Eq. (17), the nominal interest rate $i$ is a strictly decreasing function of $q$ around the Friedman rule. This is because $u^{\prime}\left(q^{*}\right) / c^{\prime}\left(q^{*}\right)=1$ and then,

$$
\left.\frac{d i}{d q}\right|_{q=q^{*}}=\left.\sigma \pi\left(e^{*}\right) \frac{d}{d q} \frac{u^{\prime}(q)}{c^{\prime}(q)}\right|_{q=q^{*}}<0 .
$$


Thus we can take a sufficiently small constant $I>0$ such that for any $i \in[0, I]$, there exists a quantity $q(i)$ that solves Eq. (17) and satisfies $q(0)=q^{*}$ and $q^{\prime}(i)<0$. From Lemma 1, we have the following:

$$
\left.\frac{d e[q(i)]}{d i}\right|_{i=0}=e^{\prime}\left(q^{*}\right) q^{\prime}(0)>0
$$

Thus the equilibrium effort $e=e(q)$ is a strictly increasing function of $i$ and a deviation from the Friedman rule increases the frequency of trades. This is what Liu et al. (2009) refer to as the hot-potato effect of inflation.

\section{Sub-optimality of the Friedman rule}

In this section, we investigate whether the Friedman rule maximizes equilibrium welfare and output.

\subsection{Inefficiency}

First, we check allocation efficiency under the Friedman rule. Steady state welfare $\pi(e) s(q)-\psi(e)$ depends on both search intensity and quantity. ${ }^{3}$ The values of $e$ and $q$ that unconditionally maximize welfare, say $e_{F B}$ and $q_{F B}$ satisfy $\pi^{\prime}(e) s(q)=\psi^{\prime}(e)$ and $s^{\prime}(q)=0$. Thus

$$
\begin{aligned}
q_{F B} & =q^{*}, \\
\frac{\psi^{\prime}\left(e_{F B}\right)}{\pi^{\prime}\left(e_{F B}\right)} & =s\left(q^{*}\right) .
\end{aligned}
$$

The function $\psi^{\prime}(e) / \pi^{\prime}(e)$ is a strictly increasing function of $e$ and satisfies $\psi^{\prime}(0) / \pi^{\prime}(0)=0$ and $\psi^{\prime}(\infty) / \pi^{\prime}(\infty)=\infty$. Thus, $e_{F B}$ exists and is uniquely determined.

Eq. (19) implies that under the Friedman rule, trades are efficient. However, the search intensity $e^{*}$ may not be efficient. From Eq. (14), $e^{*}$ satisfies the following:

$$
\frac{e^{*} \psi^{\prime}\left(e^{*}\right)}{\pi\left(e^{*}\right)}=s_{b}\left(q^{*}\right)
$$

\footnotetext{
${ }^{3}$ Strictly speaking, the stationary welfare is given by $\left[\sigma\{\pi(e) s(q)-\psi(e)\}+U\left(x^{*}\right)-x^{*}\right] /(1-\beta)$. Here we ignore the constant terms.
} 
As Eqs. (20) and (21) indicate, $e^{*}$ may differ from $e_{F B}$. Especially if $\pi(e)$ is linear, we always have $e^{*}<e_{F B}$ since $s_{b}\left(q^{*}\right)<s\left(q^{*}\right)$.

\subsection{Suboptimality}

We can express equilibrium welfare $\mathcal{W}(q)=\pi[e(q)] s(q)-\psi[e(q)]$ as a function of $q$. Since $q^{\prime}(i)<0$, a deviation from the Friedman rule improves welfare if $\mathcal{W}^{\prime}\left(q^{*}\right)<0$.

Proposition 2 A deviation from the Friedman rule improves welfare if the following holds:

$$
\epsilon_{\pi}\left(e^{*}\right)>\frac{s_{b}\left(q^{*}\right)}{s\left(q^{*}\right)}
$$

where $\epsilon_{\pi}(e)=e \pi^{\prime}(e) / \pi(e)$ is the elasticity of $\pi(e)=\zeta(e, 1)$.

Proof. See Appendix.

From Lemma 1, the social surplus is greater than the buyer's surplus and then $s_{b}(q) / s(q)<1$. Thus Eq. (22) holds if the elasticity of $\pi, \epsilon_{\pi}$ is sufficiently close to one.

The suboptimality of the Friedman rule is caused by inefficiency in search intensity. If the intensity is inefficiently low at the Friedman rule, inflation away from the rule improves welfare since the search effort increases with inflation. The search intensity is also inefficient in LR, but the first order effect of a deviation from the Friedman rule on intensity is zero, because the buyer's surplus coincides with the social surplus. Therefore, the Friedman rule is optimal in LR. However, in our model, the first order effect of inflation on search intensity is strictly positive.

We now follow LR and parametrize the model by supposing $\psi(e)=A\left(\frac{e}{1-e}\right)^{\rho}, u(q)=$ $\frac{q^{1-\theta}}{1-\theta}, c(q)=\frac{q^{1+\chi}}{1+\chi}$ and $\pi(e)=e(1-\exp (-\eta / e))$ with $A>0, \rho>1, \theta \in(0,1), \chi>0$ and $\eta>0$. In this case, equilibrium is uniquely determined if the following equality holds (see Appendix for proof) :

$$
\rho(\theta+\chi)>1+\chi
$$

The following proposition describes a condition on the desirability of the positive nominal interest rate. 
Proposition 3 If the parameters satisfy the following:

$$
A \eta^{\rho-1}>2\left(1+\frac{1}{\chi}\right)^{\rho-1}\left(\frac{\theta}{1-\theta}\right)^{\rho}
$$

then Eq. (22) holds and the Friedman rule is not the optimal monetary policy.

Proof. See Appendix.

Figure 1 depicts welfare as a function of the nominal interest rate $i$ when $A=2$, $\rho=3 / 2, \theta=1 / 2, \chi=1$, and $\eta=+\infty$. These assumptions satisfy Eq. (23).

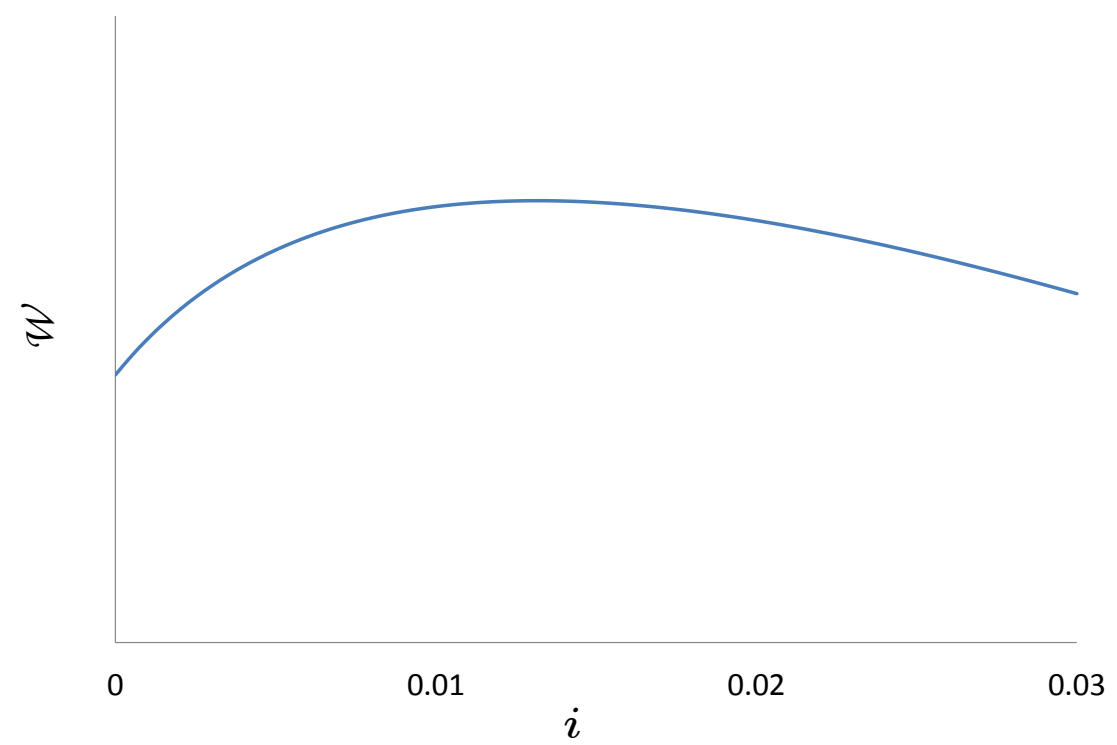

Figure 1: Inflation and welfare

Since deviation from the Friedman rule increases search intensity $e$, it may also raise output $Y=\pi(e) q$. Since $d q / d i<0$, inflation away from the Friedman rule increases output if the product of the elasticities of $\pi(e)$ and $e(q)$ is less than -1 . Figure 2 illlustrates the relationship between $Y, q$ and $i$ (the parametric assumption is the same as in Figure 1 and output under the Friedman rule is normalized to one). As evident from the figure, a positive nominal interest rate $i$ reduces $q$, but it increases $Y$ as long as $i$ is small. 


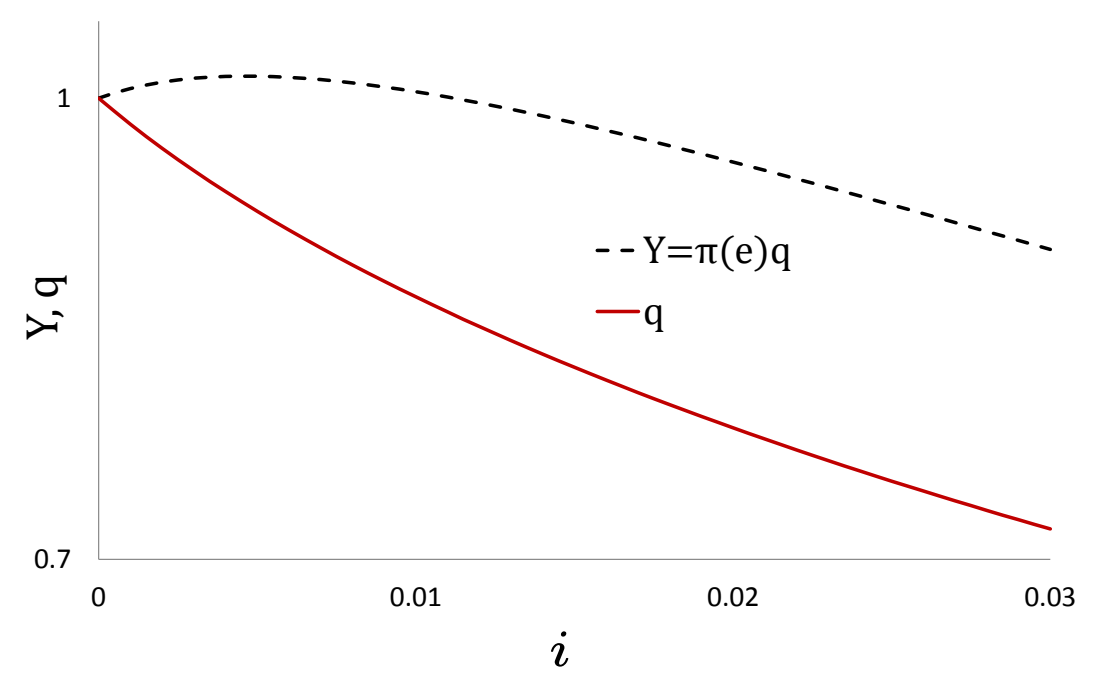

Figure 2: Output and inflation

\section{Conclusion}

We study a microfounded model of money with endogenous search intensity. In doing so, we find that if the pricing mechanism in the decentralized market is price-taking, a deviation from the Friedman rule may increase search efforts and therefore improve social welfare. This result differs from that of LR which indicates the optimality of the Friedman rule by assuming that the pricing mechanism is either bargaining or price-posting. In addition, we suggest that deviation from the Friedman rule may increase output. 


\section{Appendix}

In the Appendix, we provide proofs for propositions and a derivation of Eq. (23).

\section{A Proof of Proposition 1}

First we show Eq. (13). If $u^{\prime}(m / p)>\phi p$, then $q=m / p$ and $s_{b}^{m}=u(m / p)-\phi m$. Thus $\partial s_{b}^{m} / \partial m=\phi\left\{u^{\prime}(q) / c^{\prime}(q)-1\right\}$. If $e=\bar{e}$, then $e \bar{\alpha}=\pi(e)$ and we obtain Eq. (13). If $u^{\prime}(m / p) \leq \phi p$, then $u^{\prime}(q)=\phi p$ and $q=q^{*}$. Thus $\partial s_{b}^{m} / \partial m=0$. From Eq. (12), $i=0$. Since $q=q^{*}, u^{\prime}\left(q^{*}\right) / c^{\prime}\left(q^{*}\right)-1=0$ and Eq. (13) still holds. Next, substituting Eq. (6) into Eq. (9) yields Eq. (14). Third, Eq. (15) is the same as Eq. (6). Finally, the growth rate of the price of money is equal to the inverse of money growth rate at the stationary equilibrium; thus we obtain Eq. (16).

\section{B Proof of Lemma 1}

If $q=0, s_{b}^{\prime}(0)=u^{\prime}(0)=+\infty$ and $s_{b}^{\prime}\left(q^{*}\right)=-q^{*} c^{\prime \prime}\left(q^{*}\right)<0$. The function $\hat{c}(q) \equiv q c^{\prime}(q)-c(q)$ satisfies $\hat{c}(0)=0$ and $\hat{c}^{\prime}(q)=q c^{\prime \prime}(q)>0$. Thus $s(q)-s_{b}(q)=\hat{c}(q)>0$.

\section{Proof of Proposition 2}

Since $s^{\prime}\left(q^{*}\right)=0, \mathcal{W}^{\prime}\left(q^{*}\right)=e^{\prime}\left(q^{*}\right) \psi^{\prime}\left(e^{*}\right)\left\{\pi^{\prime}\left(e^{*}\right) s\left(q^{*}\right) / \psi^{\prime}\left(e^{*}\right)-1\right\}$. From Eq. (21), we get $\pi^{\prime}\left(e^{*}\right) / \psi^{\prime}\left(e^{*}\right)=\epsilon_{\pi}\left(e^{*}\right) / s_{b}\left(q^{*}\right)$. Thus

$$
\left.\frac{d \mathcal{W}}{d i}\right|_{i=0}=\left.e^{\prime}\left(q^{*}\right) \psi^{\prime}\left(e^{*}\right)\left(\epsilon_{\pi}\left(e^{*}\right) \frac{s\left(q^{*}\right)}{s_{b}\left(q^{*}\right)}-1\right) \frac{d q}{d i}\right|_{i=0} .
$$

Since $e^{\prime}\left(q^{*}\right)<0,\left.\frac{d \mathcal{W}}{d i}\right|_{i=0}>0$ if Eq. (22) holds.

\section{Proof of Eq. (23)}

Eqs. (13) and (14) are expressed as follows: 


$$
\begin{aligned}
i & =\sigma \pi(e)\left(q^{-\theta-\chi}-1\right), \\
\frac{(1-\theta) \rho A e^{\rho}}{\pi(e)(1-e)^{\rho+1}} & =\frac{q^{1-\theta}}{1-\theta}-q^{1+\chi}
\end{aligned}
$$

From Eq. (25), $q=\left(\frac{\pi}{\pi+i^{*}}\right)^{1 /(\chi+\theta)}$ with $i^{*}=i / \sigma$. Substitution of this equation into Eq. (26) yields the following:

$$
\frac{(1-\theta) \rho A e^{\rho}}{\pi(e)(1-e)^{\rho+1}}=\frac{\theta \pi(e)+i^{*}}{\pi(e)+i^{*}} \frac{\pi(e)^{\frac{1-\theta}{\chi+\theta}}}{\left(\pi(e)+i^{*}\right)^{\frac{1-\theta}{\chi+\theta}}} .
$$

The above equation holds when $e=0$. However, equilibrium effort level cannot equal zero. Moreover, $\pi(0)=0$ and $q=0$. If such an equilibrium exists, the marginal increase of the matching probability for the buyer is $\bar{\alpha}=\zeta(1, \infty)=1$. Therefore, for the buyer, the marginal benefit of matching with a seller is strictly positive. Since the marginal cost of effort is zero when $e=0$, the buyer does not choose $e=0$. Below, suppose $e>0$. In this case, we divide both sides of the above equation by $\pi(e)^{\frac{1-\theta}{x+\theta}}$, and we obtain the following:

$$
(1-\theta) A \rho \frac{e^{\rho-\frac{1+\chi}{\chi+\theta}}}{(1-e)^{\rho+1}}\left\{\frac{e}{\pi(e)}\right\}^{\frac{1+\chi}{\chi+\theta}}=\frac{\theta \pi(e)+i^{*}}{\pi(e)+i^{*}} \frac{1}{\left(\pi(e)+i^{*}\right)^{\frac{1-\theta}{\chi+\theta}}} .
$$

Let $g_{1}(e)$ and $g_{2}(e)$ denote the left hand side and the right hand side of Eq. (27), respectively. Since $\pi^{\prime}>0$ and $\theta<1, g_{2}^{\prime}(e)<0$. The function $e / \pi(e)$ is an increasing function; therefore, $g_{1}^{\prime}>0$ if $\rho>\frac{1+\chi}{\chi+\theta}$. In this case, $g_{1}(0)=0, g_{1}(1)>0, g_{2}(0)>0$, and $g_{2}(1)=0$. Thus, there exists a unique $e>0$ satisfying $g_{1}(e)=g_{2}(e)$.

\section{E Proof of Proposition 3}

We have $q^{*}=1, s_{b}\left(q^{*}\right)=\frac{\theta}{1-\theta}$ and $s\left(q^{*}\right)=\frac{\chi+\theta}{(1-\theta)(1+\chi)}$. The elasticity of $\pi$ is $\epsilon_{\pi}=1-$ $\frac{\eta / e}{\exp (\eta / e)-1}$. Since $s_{b}\left(q^{*}\right) / s\left(q^{*}\right)=1-\frac{\chi(1-\theta)}{\chi+\theta}$, Eq. (22) holds if

$$
\frac{\exp \left(\eta / e^{*}\right)-1}{\left(\eta / e^{*}\right)}>\frac{\theta+\chi}{\chi(1-\theta)}=1+\frac{\theta(1+\chi)}{\chi(1-\theta)}
$$

One has $\exp (x)-1>x+0.5 x^{2}$ and Eq. (28) holds if

$$
\frac{\eta}{e^{*}}>2 \frac{\theta(1+\chi)}{\chi(1-\theta)}
$$


Since $s_{b}\left(q^{*}\right)=\theta /(1-\theta)$, Eq. (14) implies the following:

$$
A\left(e^{*}\right)^{\rho-1} \frac{1-\theta}{\theta}=\left\{1-\exp \left(-\frac{\eta}{e^{*}}\right)\right\}\left(1-e^{*}\right)^{\rho+1}<1 .
$$

Then, $1 / e^{*}>\left(A \frac{1-\theta}{\theta}\right)^{1 /(\rho-1)}$. Thus, if Eq. (24) holds,

$$
\frac{\eta}{e^{*}}>\left(A \frac{1-\theta}{\theta}\right)^{1 /(\rho-1)} \eta>2 \frac{\theta(1+\chi)}{\chi(1-\theta)},
$$

and Eq. (29) holds.

\section{References}

[1] Aiyagari, R. and N. Wallace, "Existence of Steady States with Positive Consumption in the Kiyotaki-Wright Model," Review of Economic Studies 58 (1991), 901-916.

[2] Aruoba, S. B. and S. Chugh, "Optimal Fiscal and Monetary Policy when Money is Essential," Journal of Economic Theory 145 (2010), 1618-1647.

[3] Berentsen, A., G. Rocheteau, and S. Shi, "Friedman meets Hosios: Efficiency in Search Models of Money, " Economic Journal 117 (2007), 174-195.

[4] Berentsen, A., G. Camera, and C. Waller, "Money, Credit and Banking," Journal of Economic Theory 135 (2007), 171-195.

[5] Ferraris, L. and M. Watanabe, "Collateral Constraints in a Monetary Economy," Journal of Economic Theory 143 (2008), 405-424.

[6] Kiyotaki, N. and R. Wright, "On Money as a Medium of Exchange," Journal of Political Economy 97 (1989), 927-954.

[7] Kiyotaki, N. and R. Wright, "A Search-Theoretic Approach to Monetary Economics," American Economic Review 83 (1993), 63-77.

[8] Lagos, R. and G. Rocheteau, "Inflation, Output, and Welfare," International Economic Review 46 (2005), 495-522. 
[9] Lagos, R. and R. Wright, "A Unified Framework for Monetary Theory and Policy Analysis," Journal of Political Economy 113 (2005), 463-484.

[10] Liu, Q.L., S. Wang and R. Wright, "On the "Hot Potato Effect" of Inflation: Intensive versus Extensive Margins,” Macroeconomic Dynamics 15 (2011), 191-216.

[11] Rocheteau, G. and R. Wright, "Money in Search Equilibrium, in Competitive Equilibrium, and in Competitive Search Equilibrium," Econometrica 73 (2005), 175-202.

[12] Trejos, A. and R. Wright, "Search, Bargaining, Money, and Prices," Journal of Political Economy 103 (1995), 118-141.

[13] Williamson, S. and R. Wright, "New Monetarist Economics: Models," in B. Friedman and M. Woodford, eds., Handbook of Monetary Economics (Amsterdam: Elsevier, 2011), 25-96. 